\title{
Review
}

\section{Mechanism of action for deep brain stimulation and electrical neuro-network modulation (ENM)}

\author{
Michael S. Okun, M.D. ${ }^{1} *$ and Genko Oyama, M.D., Ph.D. ${ }^{12)}$
}

\begin{abstract}
Deep brain stimulation (DBS) has become an important treatment option for carefully screened medication resistant neurological and neuropsychiatric disorders. DBS therapy is not always applied deep to the brain; does not have to be applied exclusively to the brain; and the mechanism for DBS is not simply stimulation of structures. The applications and target locations for DBS devices are rapidly expanding, with many new regions of the brain, spinal cord, peripheral nerves, and muscles now possibly accessed through this technology. We will review the idea of "electrical neuro-network modulation (ENM)"; discuss the importance of the complex neural networks underpinning the effects of DBS; discuss the expansion of brain targets; discuss the use of fiber based targets; and discuss the importance of tailoring DBS therapy to the symptom, rather than the disease.
\end{abstract}

(臨床神経 2013;53:691-694)

Key words : deep brain stimulation, network, mechanism, oscillation, circuitry

\section{Introduction}

Deep brain stimulation (DBS) has become an important treatment option for carefully screened medication resistant neurological and neuropsychiatric disorders ${ }^{122}$. As the rationale and technical approaches for performing DBS improve, the therapy has rapidly evolved.

DBS was born as a natural extension of ablative brain procedures. The introduction of stereotactic surgery facilitated the placement of small and very accurate lesions to be applied deep within the human brain. These lesions were positioned first without, and later with physiological guidance. The era of ablation, which has also been referred to as lesion therapy, has included widely popular procedures such as pallidotomy and thalamotomy ${ }^{3}$. The addition of physiological guidance to existing stereotactic technique also facilitated the application of a remarkable millimeter scale accuracy ${ }^{3)}$. This level of accuracy can now be applied to many brain targets. Worldwide, lesions have been utilized for many decades to address various symptoms of neurological disorders (e.g. tremor, bradykinesia, rigidity $)^{2(4) 5)}$. One major advantage of DBS over lesion based therapies, has been its "reversibility and programmability")" DBS also has, in general, been applied to both sides of the brain, without the resultant and severe pseudobulbar complications that have been frequently observed following bilateral pallidotomy or thalamotomy approaches ${ }^{3 / 6) 7)}$.

DBS therapy has however, not been limited to the brain. The applications and locations of DBS devices have rapidly expanded, and currently the technology can be applied to the central, peripheral, autonomic, and also to the nervous system coverings ${ }^{8) ~ 11)}$. Virtually all regions of the brain, spinal cord, peripheral nerves, muscles or their coverings can ultimately be accessed, with the largest limitation being the development of a reasonable rationale, and a favorable risk/benefit ratio. DBS has not however, provided a pill free existence for most patients ${ }^{2}$, though it has facilitated profound and meaningful life improvements for over 100,000 individuals.

We don't fully understand and appreciate the underpinnings of the positive effects of DBS. In the early years of the therapy, many experts proposed that the applied electricity in some way stimulated the brain, and this stimulation evoked the positive, and sometimes dramatic benefits. Great scientific debates have since occurred on the topic of mechanisms. The debates have included champions for both inhibitory, and alternatively excitatory hypotheses. Though the mechanisms of DBS are not completely understood, the evolution of a better understanding of the complex basal ganglia circuitry has driven the powerful

*Corresponding author: UF Center for Movement Disorders \& Neurorestoration〔3450 Hull Road, 4th Floor, Gainesville, FL 32607〕

${ }^{1)}$ Center for Movement Disorders and Neurorestoration, Departments of Neurology and Neurosurgery, University of Florida

${ }^{2}$ Department of Neurology, Juntendo University, School of Medicine

(Received: 30 December 2012) 
Table 1 Summary of currently available electrical neuro-network modulation (ENM) targets and symptoms

\begin{tabular}{ll}
\hline Target(s) & Disease/symptoms may be addressed \\
\hline Brain & \\
Motor cortex & Pain, epilepsy \\
Subcallosal cingulate gyrus & Depression \\
Entorhinal cortex & Memory loss \\
Hippocampus & Memory loss \\
Subthalamic nucleus & Parkinson's disease, dystonia, tremor \\
Globus pallidus internus & Parkinson's disease, dystonia, tic \\
Thalamus & Tremor, tic \\
Hypothalamus & Cluster headache, obesity \\
Posterior subthalamic area / Zona incerta & Tremor \\
Spinal cord & Pain \\
Peripheral nerve & \\
Vagus nerve & Epilepsy, pain \\
Trigeminal never & Epilepsy, pain \\
Muscle & Muscle weakness \\
\hline
\end{tabular}

notion that DBS drives a larger neural network ${ }^{12) \sim 16)}$.

We will briefly review and address "electrical neuro-network modulation (ENM)", and we will discuss the importance of a complex network in explaining mechanism of action of DBS. We will also address what is known about the potential mechanisms of action, the expansion of target and fiber sites, and the notion that tailoring the therapy to the symptom, rather than the disease, will be important for the development of a personalized medicine approach to DBS.

\section{The electric brain and beyond}

Currently, the term DBS refers to targets stimulated "deep" within the brain tissue. If the field persists in referring to the therapy as DBS, we must define what is meant by "deep"? Definitions excluding the cortex and/or the brain coverings would mean that the term DBS could not be used to describe electricity applied to these other areas. Also, the term DBS could not be applied to direct stimulation of the brain's many superficially located subcortical fiber bundles. These fiber bundles will likely make up the next generation of rational electrical stimulation targets. The term deep is therefore imprecise.

Beyond the brain and its coverings, there are many other potential nervous system modulation targets, all of which are potentially amenable to an electrical therapy ${ }^{210) 11(17) \sim 21)}$. If we continue to use the term DBS to exclusively refer to very deep brain targets, then we will be forced to coin new terminology to describe stimulation for every specific central, peripheral, and autonomic nervous system region. This approach has its merits. For example, referring to motor cortex stimulation, spinal cord stimulation, and specific nerve stimulation (i.e. vagus nerve stimulation) will ultimately result in less ambiguity about what is actually being stimulated. However, with the explosion of potential new DBS targets this approach may not be feasible, and an umbrella term may thus become necessary to describe the breadth of the field.

The general term modulation can be applied to many pharmacological, behavioral, gene therapy, neurotrophic factor, or other approaches, inclusive of electrical methodologies. Therefore, using the term modulation or neuromodulation to apply only to the application of electricity is similar to using the term deep; it is imprecise. Electrical modulation, or alternatively ENM would provide a more accurate description of the therapy and would more broadly cover its potential mechanisms of action.

\section{Current thinking on the mechanisms of action}

A great transoceanic debate occurred in the 1990's over the mechanisms underpinning the therapeutic effects of DBS. Initially an inhibitory hypothesis was put forth by the founding French group, with the predominant thought that the most likely mechanism of action was jamming (retrograde activation of axons) of the electrical circuit ${ }^{22}$. Later it was proposed that DBS acted in a similar manner to lesion therapy, however this notion was also disproven ${ }^{12) \sim 14) 22)}$

Myelinated axons, cell bodies and dendrites are all known to have various and different electrical properties. The appreciation of these properties has led to finite modeling of DBS currents, and to a serious challenge to the inhibitory hypothesis ${ }^{12) 14}$ ). The modeling has led to a better appreciation of the important role of axons in the underpinnings of DBS. One recent provocative theory has been that DBS acts as an "informational 


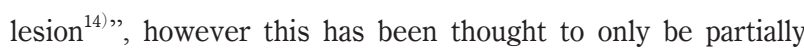
accurate.

It is widely appreciated that there can be neurotransmitter fatigue, and excitation of axons. One intriguing aspect of DBS is that the activity in the axon and in the cell body can be decoupled. Additionally DBS can act on astrocytes to trigger a propagating calcium wave, glial activation, neurotransmitter release and subsequent neuromodulation, based not only on electrical influences, but also chemical influences. In total we now appreciate that DBS changes the rate, and also the firing pattern of neurons (i.e. disrupts the disruption), DBS can be both excitatory and inhibitory, DBS can affect local blood flow, and DBS can possibly stimulate neurogenesis ${ }^{12) \sim 16) 22) 23)}$. Thus, one may reasonably conclude that the mechanisms underpinning DBS are more than just "stimulation".

The idea that applying electricity can lead to rapid changes in complex motor, mood, and behavioral symptoms raises the intriguing possibility that DBS acts on a larger neural network ${ }^{12) 23)}$. This idea has been bolstered by the discovery of the family of parallel basal ganglia circuits ${ }^{24) 25}$, and also supported by the idea that DBS changes network-wide oscillations and that there may be coherence between subcortical and cortical brain signals ${ }^{26) 27)}$. The later hypothesis has led to the rapid development of efforts aimed at unlocking signature brain patterns for various motor, mood, and cognitive changes, and to looking at electrical corticography.

\section{Tailoring electrical neuro-network modulation}

The introduction of DBS has led to an expanding list of potential indications such as Parkinson's disease, tremor, dystonia, epilepsy, cluster headache, memory loss, obsessive compulsive symptoms, tics, and depression ${ }^{1 / 9) 20)}$. Unfortunately, the indications for DBS therapy are usually referred to by disease states, rather than by disease symptoms. As DBS evolves, and we better understand its potential(s), discussions with patients should shift from disease states to specific symptoms. Doctors and interdisciplinary teams charged with selecting patients should not be as concerned with the underlying neurological or neuropsychiatric disorder, as with the specific set of symptoms that are disabling, and potentially addressable for an individual patient. Tailoring the therapy to focus on specific symptoms will be an important evolution for the field of ENM (Table 1$)^{2)}$.

\section{The future of electrical neuro-network modulation}

The immediate future of ENM will include an evolution toward symptom specific tailoring ${ }^{2)}$. There will undoubtedly be an expansion of available targets, likely with the addition of fiber bundle targeting (FBT), and more specific targeting of neuronal and non-neuronal elements. The therapy will become more open to the use of multiple leads, and also to rescue leads ${ }^{28) 29}$, especially as new symptoms emerge across various disease states. The hardware will become smaller, and neurostimulator placement in the chest (with connector wires) will disappear, thus dropping infection rates, erosions, and consequently improving the morbidity rate of the procedure. Devices will become smaller and smarter. Improved understanding of single cell and local field potential physiology will facilitate monitoring of the brain's inherent electrical signatures for various motor, cognitive and behavioral manifestations. Doctors, nurses, and programmers will be able to monitor and to control devices remotely. Patients will be granted more access to their personal electrical settings, and they will be granted the ability to tune their own device "in a situation dependent fashion". Telemedicine will be utilized to improve satisfaction, and to alleviate access problems for those with financial and/or travel constraints. Collectively all of these advances will lead to more efficient and more rational device programming, and to higher overall patient satisfaction. Additionally, ENM has great potential to be coupled to other therapies in the near-future (gene therapy, neurotrophic factors, stem cells, etc.). Understanding the mechanisms of this therapy has the potential to unlock and to inform more rational pharmacological, and other novel therapeutic approaches.

In summary, the term DBS is imprecise. It is not always deep, not always brain, and not always stimulation. The use of other terms such as modulation and neuromodulation are also imprecise, as these terms can be applied to pharmacological, and other therapeutic approaches. The weight of the evidence suggests that DBS therapy involves changes across a complex neural network. Therefore, currently, what is referred to as DBS therapy, can be more precisely described by the umbrella term, electrical neuro-network modulation (ENM), and as our understanding expands on the mechanisms of action of DBS we should keep idea in mind that the network concept is critical.

Acknowledgements: The author would like to acknowledge the critical input provided by David Riley from Case Western University.

※ The authors declare there is no conflict of interest relevant this article.

\section{References}

1) Benabid AL, Chabardes S, Torres N, et al. Functional neurosurgery for movement disorders: a historical perspective. Prog Brain Res 2009;175:379-391.

2) Okun MS, Foote KD. Parkinson's disease DBS: what, when, who and why? The time has come to tailor DBS targets. Expert Rev Neurother 2010;10:1847-1857.

3) Okun MS, Vitek JL. Lesion therapy for Parkinson's disease and 
other movement disorders: update and controversies. Mov Disord 2004;19:375-389.

4) Baron MS, Vitek JL, Bakay RA, et al. Treatment of advanced Parkinson's disease by posterior GPi pallidotomy: 1-year results of a pilot study. Ann Neurol 1996;40:355-366.

5) Bronstein JM, Tagliati M, Alterman RL, et al. Deep brain stimulation for Parkinson disease: an expert consensus and review of key issues. Arch Neurol 2011;68:165.

6) Gross RE, Lozano AM, Lang AE, et al. The effects of pallidotomy on Parkinson's disease: study design and assessment techniques. Acta Neurochir Suppl 1997;68:24-28.

7) Laitinen LV, Bergenheim AT, Hariz MI. Leksell's posteroventral pallidotomy in the treatment of Parkinson's disease. J Neurosurg 1992;76:53-61.

8) Benabid AL. What the future holds for deep brain stimulation. Expert Rev Med Devices 2007;4:895-903.

9) Bernstein AJ, Peters KM. Expanding indications for neuromodulation. Urol Clin North Am 2005;32:59-63.

10) Daneshgari F, Moy ML. Current indications for neuromodulation. Urol Clin North Am 2005;32:37-40.

11) Levy R, Deer TR, Henderson J. Intracranial neurostimulation for pain control: a review. Pain Physician 2010;13:157-165.

12) McIntyre CC, Hahn PJ. Network perspectives on the mechanisms of deep brain stimulation. Neurobiol Dis 2010;38:329337.

13) Dostrovsky JO, Lozano AM. Mechanisms of deep brain stimulation. Mov Disord 2002;17 Suppl 3:S63-68.

14) Grill WM, Snyder AN, Miocinovic S. Deep brain stimulation creates an informational lesion of the stimulated nucleus. Neuroreport 2004;15:1137-1140.

15) Grill WM, Cantrell MB, Robertson MS. Antidromic propagation of action potentials in branched axons: implications for the mechanisms of action of deep brain stimulation. J Comput Neurosci 2008;24:81-93.

16) Lozano AM, Mahant N. Deep brain stimulation surgery for Parkinson's disease: mechanisms and consequences. Parkinsonism Relat Disord 2004;10 Suppl 1:S49-57.
17) Visser-Vandewalle V, Temel Y, van der Linden C, et al. Deep brain stimulation in movement disorders. The applications reconsidered. Acta Neurol Belg 2004;104:33-36.

18) Mohr P. Deep brain stimulation in psychiatry. Neuro Endocrinol Lett 2008;29 Suppl 1:123-132.

19) Luigjes J, van den Brink W, Feenstra $M$, et al. Deep brain stimulation in addiction: a review of potential brain targets. Mol Psychiatry 2012;17:572-583.

20) Lozano AM, Hamani C. The future of deep brain stimulation. J Clin Neurophysiol 2004;21:68-69.

21) Krack P, Hariz MI, Baunez C, et al. Deep brain stimulation: from neurology to psychiatry? Trends Neurosci 2010;33:474484.

22) Benabid AL, Koudsie A, Benazzouz A, et al. Subthalamic stimulation for Parkinson's disease. Arch Med Res 2000;31:282-289.

23) Vedam-Mai V, van Battum EY, Kamphuis W, et al. Deep brain stimulation and the role of astrocytes. Mol Psychiatry 2012;17: 124-131.

24) Delong MR, Georgopoulos AP, Crutcher MD, et al. Functional organization of the basal ganglia: contributions of single-cell recording studies. Ciba Found Symp 1984;107:64-82.

25) Alexander GE, DeLong MR, Strick PL. Parallel organization of functionally segregated circuits linking basal ganglia and cortex. Ann Rev Neurosci 1986;9:357-381.

26) Litvak V, Jha A, Eusebio A, et al. Resting oscillatory corticosubthalamic connectivity in patients with Parkinson's disease. Brain 2011;134:359-374.

27) Wagle Shukla A, Okun MS. Personalized medicine in deep brain stimulation through utilization of neural oscillations. Neurology 2012;78:1900-1901.

28) Ellis TM, Foote KD, Fernandez HH, et al. Reoperation for suboptimal outcomes after deep brain stimulation surgery. Neurosurgery 2008;63:754-760.

29) Oyama G, Foote KD, Hwynn N, et al. Rescue leads: a salvage technique for selected patients with a suboptimal response to standard DBS therapy. Parkinsonism Related Disord 2011;17: 451-455. 